\title{
ASPECTOS DA BIOLOGIA DE Aphis gossypii Glover, 1877 (Hemiptera: Aphididae) EM QUATRO CULTIVARES DE ALGODOEIRO, EM LABORATÓRIO
}

\author{
Biological aspects of Aphis gossypii Glover, 1877 \\ (Hemiptera: Aphididae) on four cotton cultivars, in laboratory
}

\author{
Luis Gustavo Amorim Pessoa ${ }^{1}$, Brígida Souza $^{2}$, César Freire Carvalho ${ }^{2}$, Marlene Gonçalves Silva ${ }^{3}$
}

\begin{abstract}
RESUMO
Objetivou-se estudar alguns aspectos biológicos de Aphis gossypii Glover alimentado nas cultivares de algodoeiro Allen, IPEACO-SL 21-61131, JPM 781-88-3 e Auburn SM 310, em experimentos conduzidos a $25 \pm 2^{\circ} \mathrm{C}$, $60 \pm 10 \%$ UR e fotofase de 12 horas, em delineamento inteiramente casualizado. Fêmeas adultas obtidas após a última ecdise foram individualizadas em discos foliares dessas cultivares durante 24 horas e das ninfas produzidas, uma foi escolhida ao acaso para condução do experimento. Avaliaram-se também a densidade e os tipos de tricomas em cada cultivar. A JPM 781-88-3 permitiu o melhor desenvolvimento do pulgão, com uma produção diária de 4,2 \pm 0,1 ninfas/fêmea e um total de 69,2 $\pm 2,1$. Na cultivar IPEACO-SL 2261131, ocorreram um prolongamento da fase ninfal e redução na fecundidade, com uma média diária de 3,0 \pm 0,3 ninfas/fêmea e um total de 54,9 \pm 2,2 ninfas/fêmea. A cultivar Auburn SM 310 afetou o ciclo biológico desse afídeo, reduzindo a sua duração em relação às demais cultivares. Com relação aos tipos de tricomas, foram identificadas estruturas não glandulares de formas estreladas e glandulares, com predominância do primeiro tipo. As maiores densidades de tricomas não glandulares foram observadas nas cultivares Allen e IPEACO-SL 21-61131, enquanto os glandulares ocorreram em maior número nas cultivares JPM 78188-3 e Allen. Na Auburn SM 310, não foi encontrado nenhum tipo de tricoma.
\end{abstract}

Termos para indexação: Algodoeiro, tricoma, pulgão do algodoeiro.

\begin{abstract}
It was aimed to study some biological aspects of Aphis gossypii Glover fed on cotton cultivars Allen, IPEACO-SL 21-61131, JPM 781-88-3 and Auburn SM 310 in experiments conducted at $25 \pm 2^{\circ} \mathrm{C}, 60 \pm 10 \%$ of RH and 12-hour photophase in a completely randomized design. Adult females obtained after the last ecdysis were mantained on leaf disks of those cultivars for 24 hours and from the produced nymphs, one was chosen at random for the conduction of the experiment. The density and the sorts of trichomes on each cultivar were evaluated. The cultivar JPM 781-88-3 enabled the best development of the aphid with a daily production of $4.2 \pm 0.1$ nymphs/female and a total of $69.2 \pm 2.1$. On the cultivar IPEACO-SL 22-61131, a lenghtening of the nymphal stage and reduced fecundity took place with a daily mean of $3.0 \pm 0.3$ nymphs/female and a whole of $54.9 \pm 2.2$ nymphs/female. The cultivar Auburn SM 310 affected the biological cycle of this aphid, reducing its duration. As regards the sorts of trichome, star-shaped non-glandular and glandular structures were identified, with the predominance of the first type. The highest densities of non-glandular trichomes were found on the cultivars Allen and IPEACO-SL 21-61131 while the glandular ones occurred in a greater number on the cultivars JPM 781-88-3 and Allen. On Auburn SM 310 no sort of trichom was found.
\end{abstract}

INDEX TERMS: cotton, trichome, cotton aphid.

(Recebido para publicação em $1^{\circ}$ de julho de 2002 e aprovado em 13 de dezembro de 2004)

\section{INTRODUÇÃO}

O algodão é a mais importante fibra têxtil do mundo (MATTHEWS e TUNSTALL, 1994), sendo o Brasil o maior produtor do Hemisfério Sul (NEVES et al., 1965; PASSOS, 1977; BELTRÃO et al., 1986). Embora algumas características das fibras, como comprimento, espessura, resistência e maturidade, sejam hereditárias, inúmeros fatores físicos, químicos e mecânicos contribuem para alterá-las no decorrer do seu desenvolvimento. Entre esses fatores, estão os ar- trópodes-praga, os quais causam prejuízos consideráveis na produção.

Entre o complexo de pragas do algodoeiro, o pulgão Aphis gossypii Glover, 1877 (Hemiptera: Aphididae) tem se destacado pelo aumento da resistência aos inseticidas e escape à predação e ao parasitismo devido a destruição desses agentes de controle, podendo provocar reduções de até $40 \%$ na produção (PASSOS, 1977; WEATHERSBEE III e HARDEE, 1994).

1. Engenheiro Agrônomo, MSc., Departamento de Entomologia, Universidade Federal de Lavras/UFLA - Caixa Postal 3037 - $37200-000$ - Lavras, MG.

2. Professores do Departamento de Entomologia/UFLA.

3. Engenheira Agrônoma, Departamento de Entomologia/UFLA. 
Muitas características do algodoeiro podem conferir resistência às pragas, destacando-se as brácteas frego, folhas okra, caráter glabro e o hirsuto contra diversas espécies de artrópodes-praga. Busoli et al. (1994) relataram a utilização do caráter glabro e do hirsuto contra o pulgão do algodoeiro, porém, Soares (1999) definiu esses caracteres como conflitantes ou não-constatados para esse inseto.

Com este trabalho teve-se por objetivo avaliar os efeitos de quatro cultivares de algodoeiro (Gossypium hirsutum L.) sobre alguns aspectos da biologia de ninfas e adultos de A. gossypii, em condições de laboratório.

\section{MATERIAL E MÉTODOS}

Os experimentos foram conduzidos em sala climatizada a $25 \pm 2^{\circ} \mathrm{C}$, UR $60 \pm 10 \%$ e fotofase de 12 horas, com insetos provenientes de uma criação na cultivar IAC 22, existente no Departamento de Entomologia da Universidade Federal de Lavras - UFLA, MG.

Utilizaram-se folhas do terço médio das cultivares de algodoeiro Auburn SM 310, JPM 781-88-3, Allen e IPEACO-SL 22-61131, amplamente utilizadas em cultivos na região de Janaúba, no norte do Estado de Minas Gerais. Procedeu-se à desinfestação das folhas e confecção de discos foliares, que foram acondicionados em placas de Petri de $5 \mathrm{~cm}$ de diâmetro contendo uma lâmina de ágar-água de aproximadamente $5 \mathrm{~mm}$. Os discos foram dispostos com a superfície abaxial para cima, pois, segundo Treacy et al. (1987), é a face onde se concentra a maior densidade de tricomas e local preferido para o desenvolvimento de A. gossypii. As placas foram vedadas com pvc laminado perfurado com um estilete para evitar condensação no interior, e colocadas de forma invertida, simulando a situação que ocorre em condições naturais. Os discos foram substituídos à medida que se apresentavam com a coloração amarelada e sinais de ressecamento.

Com o auxílio de um pincel fino, foi colocada uma fêmea adulta, obtida após a última ecdise ninfal, em cada disco foliar. Após 24 horas, a fêmea foi retirada e, das ninfas produzidas, uma foi escolhida para a condução do experimento. Foram feitas observações a cada 24 horas avaliando-se a duração de cada ínstar e do período ninfal, viabilidade das ninfas, duração do período reprodutivo e do ciclo biológico e capacidade de multiplicação. Para manutenção do mesmo número de repetições na fase adulta, conduziu-se uma criação paralela, nas mesmas condições experimentais, para eventuais reposições nos casos de mortalidade ninfal.
Utilizou-se o delineamento experimental inteiramente casualizado com quatro tratamentos e dez repetições, cada uma constituída por nove placas de Petri contendo um disco foliar com um afídeo, totalizando 90 discos por cultivar. Os dados porcentuais referentes à viabilidade foram transformados para arco-seno da $\sqrt{X}$ e aqueles referentes à duração dos ínstares, período ninfal, produção diária e total de ninfas, longevidade e ciclo biológico foram transformados para $\sqrt{X}$. Efetuou-se a análise de variância e aplicou-se o teste de agrupamento de médias de Scott e Knott a 5\% de probabilidade.

Visando a complementar o estudo, avaliaram-se o tipo e a densidade de tricomas em folhas retiradas do terço médio de plantas com altura média de $30 \mathrm{~cm}$. Foram efetuados cortes paradérmicos na face abaxial das folhas, os quais foram montados em lâmina e corados com safranina $0,1 \%$ em água + glicerina. Utilizou-se o mesmo delineamento experimental e número de repetições do ensaio anterior e, nesse caso, cada repetição foi constituída por seis cortes. Os dados referentes à contagem dos tricomas foram transformados para $\sqrt{X}$, submetidos à análise de variância e comparados pelo teste de agrupamento de médias de Scott e Knott a 5\% de probabilidade.

\section{RESULTADOS E DISCUSSÃO}

Foram identificados dois tipos de tricomas: não glandular ramificado, de forma estrelada, e glandular com pedúnculo curto e cabeça multicelular, sendo observadas diferenças significativas no número dessas estruturas em função do tipo e das cultivares de algodoeiro (Tabela 1).

As cultivares Allen e IPEACO-SL 22-61131 apresentaram as maiores densidades de tricomas não glandulares e as cultivares JPM 781-88-3 e Allen apresentaram as maiores densidades de tricomas glandulares. A Auburn SM 310 não apresentou nenhum tipo de tricoma, porém, nas demais, os não glandulares foram significativamente mais numerosos que os glandulares. Vários estudos têm demonstrado a associação entre graus de pubescência em plantas de algodão e a resistência a determinados insetos-praga, porém, diferentes respostas relacionadas, não somente a herbívoros, mas também a inimigos naturais, evidenciam a necessidade de maiores conhecimentos envolvendo a interação desses organismos.

As ninfas de A. gossypii apresentaram quatro ínstares quando criadas em todas as cultivares, assemelhando-se às observações feitas por Vendramim e Nakano (1981), para esse afídeo criado em plantas de algodão em dois estágios fenológicos. Constatações seme-

Ciênc. agrotec., Lavras, v. 28, n. 6, p. 1235-1239, nov./dez., 2004 
lhantes foram realizadas em plantas da família Cucurbitaceae por Aldyhim e Khalil (1993) e Kocourek et al. (1994), e em crisântemo por Soglia et al. (2002).

TABELA 1 - Número de tricomas ( \pm EP), em função do tipo e das cultivares de algodoeiro, Lavras, MG. 2001.

\begin{tabular}{lcc}
\hline \multicolumn{1}{c}{$\begin{array}{c}\text { Cultivares } \\
\text { de Algodoeiro }\end{array}$} & \multicolumn{2}{c}{ Tipos de Tricomas } \\
\cline { 2 - 3 } & $\begin{array}{c}\text { Não } \\
\text { Glandulares }\end{array}$ & Glandulares \\
\hline Auburn SM 310 & $0,00 \pm 0,00 \mathrm{aC}$ & $0,00 \pm 0,00 \mathrm{aB}$ \\
JPM 781-88-3 & $7,81 \pm 0,67 \mathrm{aB}$ & $0,93 \pm 0,17 \mathrm{bA}$ \\
Allen & $14,47 \pm 1,02 \mathrm{aA}$ & $0,80 \pm 0,14 \mathrm{bA}$ \\
IPEACO-SL 22- & $15,47 \pm 0,93 \mathrm{aA}$ & $0,32 \pm 0,11 \mathrm{bB}$ \\
61131 & & \\
\hline
\end{tabular}

$\mathrm{CV}(\%)=7,90$

${ }^{1}$ Médias seguidas pela mesma letra minúscula nas linhas e maiúscula nas colunas não diferem significativamente entre si pelo teste de Scott e Knott $(P<0,05)$.

Não foi observado efeito significativo das cultivares na duração dos ínstares desse afídeo (Tabela 2). Resultados semelhantes foram encontrados por Vendramim e Nakano (1981) quando estudaram a biologia comparada das formas verdes e amarelas dessa espécie em folhas de algodoeiro da cultivar IAC 17, em casa-de-vegetação. Soglia et al. (2002), testando cultivares de crisântemo no desenvolvimento desse pulgão, em laboratório, também não observaram diferenças na duração desses estádios. Mesmo em se tratando de outras espécies vegetais, os resultados até então obtidos não têm evidenciado o efeito da pilosidade sobre o número e a duração dos ínstares de A. gossypii.

A fase ninfal de $A$. gossypii foi afetada pela cultivar IPEACO-SL 22-61131, a qual proporcionou uma maior duração desse período. Vendramim e Nakano (1981) constataram uma duração de 5,8 e 5,9 dias para a fase ninfal das formas verdes e amarelas desse pulgão, respectivamente, criado na cultivar IAC 17, em casa-de-vegetação. Em laboratório, Kocourek et al. (1994), estudando a biologia desse inseto criado em algodoeiro, obtiveram uma duração de 5,0 dias, e Aldyhim e Khalil (1993), em estudos sobre o desenvolvimento de A. gossypii em Curcubita pepo L., encontraram uma duração de 5,6 dias. Em função dessas pesquisas, verifica-se uma proximidade nos resultados, independentemente do hospedeiro e condições de condução dos experimentos.

Não foi verificado efeito das cultivares de algodoeiro sobre a viabilidade dos ínstares e da fase ninfal de A. gossypii, constatando-se uma sobrevivência compreendidada entre 93,3 e 100\% (Tabela 3). De um modo geral, a pubescência nas cultivares não afetou de modo significativo as variáveis biológicas estudadas na fase jovem dessa espécie de pulgão, sugerindo que, na densidade em que ocorre nessas cultivares, não influencia a biologia desse inseto.

TABELA 2 - Duração (dias) ( \pm EP) dos ínstares de Aphis gossypii, em função das cultivares de algodoeiro, Lavras, MG. 2001.

\begin{tabular}{lccccc}
\hline \multirow{2}{*}{$\begin{array}{c}\text { Cultivares de } \\
\text { Algodoeiro }\end{array}$} & Primeiro & Segundo & Terceiro & Quarto & $\begin{array}{c}\text { Total da } \\
\text { Fase Ninfal* }\end{array}$ \\
\cline { 2 - 5 } & $1,47 \pm 0,08$ & $1,12 \pm 0,07$ & $1,04 \pm 0,02$ & $1,21 \pm 0,05$ & $4,94 \pm 0,08 \mathrm{~B}$ \\
\hline Auburn SM 310 & $1,53 \pm 0,09$ & $1,04 \pm 0,02$ & $1,11 \pm 0,05$ & $1,24 \pm 0,06$ & $4,92 \pm 0,07 \mathrm{~B}$ \\
JPM 781-88-3 & $1,36 \pm 0,06$ & $1,03 \pm 0,02$ & $1,10 \pm 0,04$ & $1,44 \pm 0,05$ & $4,93 \pm 0,06 \mathrm{~B}$ \\
Allen & $1,56 \pm 0,07$ & $1,14 \pm 0,04$ & $1,21 \pm 0,04$ & $1,31 \pm 0,08$ & $5,23 \pm 0,09 \mathrm{~A}$ \\
IPEACO-SL 22-61131 & 12,56 & 8,62 & 3,12 & 10,78 & 4,95 \\
\hline CV (\%) & &
\end{tabular}

*Médias seguidas pela mesma letra maiúscula não diferem significativamente entre si pelo teste de Scott e Knott $(\mathbf{P}<0,05)$. 
Os resultados obtidos para a viabilidade da fase de ninfa aproximaram-se daqueles encontrados por Kersting et al. (1999) que, trabalhando em condições semelhantes e com a mesma espécie de afídeo criado em algodoeiro, obtiveram uma viabilidade de 95,7\%. Entretanto, diferiram daqueles obtidos por Soglia et al. (2002), que constataram efeito negativo das cultivares de crisântemo com densidades de tricomas variando de 16,6 a 21,6 tricomas $/ \mathrm{mm}^{2}$, sobre a sobrevivência de ninfas dessa espécie, o que foi atribuído à formação de uma barreira mecânica, dificultando a penetração dos estiletes no tecido foliar. As discrepâncias dos resultados verificados para essas duas espécies vegetais provavelmente se devem aos aspectos morfológicos, químicos e aqueles relacionados à adaptabilidade do organismo fitófago à planta hospedeira.

Com relação aos aspectos biológicos da fase adulta, verificou-se que na cultivar IPEACO-SL 22-61131 os pulgões apresentaram um maior período reprodutivo em relação às demais. Os tricomas encontrados nessa cultivar não podem ser considerados como o mecanismo causador dos efeitos adversos a esse afídeo, pois a densidade em que ocorrem não diferiu da cultivar Allen. As diferenças verificadas na duração desse período sugerem a presença de algum outro fator que não a pilosidade, possivelmente de natureza química, os quais estariam interferindo negativamente no desenvolvimento desse afídeo (Tabela 4).

As cultivares Auburn SM 310 e JPM 781-88-3 permitiram uma produção diária de ninfas significativamente maior em relação às demais, e a IPEACO-SL 22-61131 afetou de forma negativa, causando uma menor produção de ninfas. O total de ninfas produzidas ao longo do período reprodutivo também foi afetado pela cultivar de algodoeiro em que os afídeos foram criados, constatando-se uma produção significativamente maior na JPM 781-88-3, ao passo que na IPEACO-SL 22-61131, observou-se a produção de um menor número de ninfas (Tabela 4). Algum fator de natureza química pode ter sido o responsável pelo efeito negativo da cultivar IPEACO-SL 22-61131sobre a fecundidade desse pulgão.

TABELA 3 - Viabilidade (\%) (土 EP) dos ínstares e da fase ninfal de Aphis gossypii, em função das cultivares de algodoeiro, Lavras, MG. 2001.

\begin{tabular}{lccccc}
\hline \multirow{2}{*}{ Cultivares de Algodoeiro } & \multicolumn{4}{c}{ Ínstares $^{\mathbf{1}}$} & $\begin{array}{c}\text { Total da } \\
\text { Fase Ninfal }\end{array}$ \\
\cline { 2 - 5 } & Primeiro & Segundo & Terceiro & Quarto & $96,7 \pm 1,5$ \\
Auburn SM 310 & $98,9 \pm 1,1$ & $100,0 \pm 0,0$ & $100,0 \pm 0,0$ & $97,8 \pm 1,5$ & 100,0 \\
JPM 781-88-3 & $94,4 \pm 4,4$ & $100,0 \pm 0,0$ & $100,0 \pm 0,0$ & $100,0 \pm 0,0$ & $93,3 \pm 2,6$ \\
Allen & $100,0 \pm 0,0$ & $100,0 \pm 0,0$ & $100,0 \pm 0,0$ & $100,0 \pm 0,0$ & $100,0 \pm 0,0$ \\
IPEACO-SL 22-61131 & $98,9 \pm 1,1$ & $100,0 \pm 0,0$ & $98,9 \pm 1,1$ & $100,0 \pm 0,0$ & $97,8 \pm 1,4$ \\
\hline CV (\%) & 6,78 & 8,62 & 1,67 & 2,22 & 8,06 \\
\hline
\end{tabular}

${ }^{1}$ Não houve diferença significativa entre os tratamentos pelo teste $F(P<0,05)$.

TABELA 4 - Aspectos biológicos ( \pm EP) de Aphis gossypii, em função das cultivares de algodoeiro, Lavras, MG. 2001.

\begin{tabular}{lcccc}
\hline Cultivares de algodoeiro & $\begin{array}{c}\text { Período reprodutivo } \\
\text { (dias) }\end{array}$ & Ninfas/fêmea/dia & $\begin{array}{c}\text { Total de nin- } \\
\text { fas/fêmea }\end{array}$ & $\begin{array}{c}\text { Ciclo biológico } \\
\text { (dias) }\end{array}$ \\
\hline Auburn SM 310 & $15,98 \pm 0,60 \mathrm{~B}$ & $4,08 \pm 0,15 \mathrm{~A}$ & $62,03 \pm 2,99 \mathrm{~B}$ & $31,59 \pm 1,18 \mathrm{~B}$ \\
JPM 781-88-3 & $17,48 \pm 0,91 \mathrm{~B}$ & $4,16 \pm 0,13 \mathrm{~A}$ & $69,16 \pm 2,12 \mathrm{~A}$ & $37,09 \pm 0,96 \mathrm{~A}$ \\
Allen & $18,22 \pm 0,38 \mathrm{~B}$ & $3,56 \pm 0,09 \mathrm{~B}$ & $60,51 \pm 1,76 \mathrm{~B}$ & $35,60 \pm 0,92 \mathrm{~A}$ \\
IPEACO-SL 22-61131 & $20,84 \pm 1,69 \mathrm{~A}$ & $3,00 \pm 0,33 \mathrm{C}$ & $54,89 \pm 2,16 \mathrm{C}$ & $37,13 \pm 1,19 \mathrm{~A}$ \\
\hline CV (\%) & 12,19 & 13,57 & 10,85 & 7,97 \\
\hline
\end{tabular}

${ }^{1}$ Médias seguidas pela mesma letra maiúscula nas colunas não diferem significativamente entre si pelo teste de Scott e Knott $(\mathbf{P}<\mathbf{0 , 0 5})$. 
O ciclo biológico de $A$. gossypii foi afetado pela cultivar em que os insetos se desenvolveram, observando-se uma redução na sua duração para afídeos criados na cultivar Auburn SM 310, e nesse período, foram capazes de manter uma produção de ninfas comparável àquelas constatadas nas demais cultivares (Tabela 4).

Pelos resultados obtidos, não se permite afirmar que a pilosidade constitui-se em uma das causas de resistência do algodoeiro ao pulgão $A$. gossypii, uma vez que os afídeos desenvolveram-se em todas as cultivares, independentemente da presença ou ausência de tricomas. Observações semelhantes foram feitas por Soares (1999), que mencionou a pilosidade como uma característica inócua a esse afídeo. Os efeitos negativos verificados nos parâmetros biológicos avaliados durante o desenvolvimento de $A$. gossypii, tais como redução da fecundidade e alteração no tempo de vida, enquadram-se como mecanismo de resistência por antibiose, sendo esse o provável mecanismo de defesa dessas cultivares contra essa espécie de pulgão.

\section{CONCLUSÕES}

A duração da fase ninfal de A. gossypii foi afetada pelas cultivares de algodoeiro; contudo, os efeitos foram mais expressivos na fase adulta do pulgão.

A cultivar de algodoeiro JPM 781-88-3 foi a que melhor permitiu o desenvolvimento de A. gossypii, ao passo que a cultivar IPEACO SL 61-131 afetou de forma negativa a biologia desse afídeo.

\section{AGRADECIMENTOS}

Ao Professor Manuel Losada Gavilanes, Departamento de Biologia da UFLA, pela orientação no preparo dos cortes paradérmicos nas folhas de algodoeiro. Ao Banco do Nordeste do Brasil S/A-BNB e Fundação de Desenvolvimento Científico e Tecnológico da Agropecuária Norte Mineira-FUNDETEC, pelo auxílio financeiro para a execução deste trabalho.

\section{REFERÊNCIAS BIBLIOGRÁFICAS}

ALDYHIM, Y. N.; KHALIL, A. F. Influence of temperature and daylength on population development of Aphis gossypii on Curcubita pepo. Entomologia Experimentalis et Applicata, Dordrecht, v. 67, p. 167-172, 1993.

BELTRÃO, N. E. M. et al. O algodão no nordeste brasileiro e tecnologias disponíveis. Fortaleza: Centro Nacional de Pesquisa do Algodão; Banco do Nordeste do Brasil, 1986. 168 p.
BUSOLI, A. C.; SOARES, J. J.; LARA, F. M. O bicudo do algodoeiro e seu manejo. Jaboticabal: FCAV/ UNESP, 1994. 32 p.

KERSTING, U.; SATAR, S.; UYGUN, N. Effect of temperature on development rate and fecundity of apterous Aphis gossypii Glover (Homoptera: Aphididae) reared on Gossypium hirsutum L. Journal of Applied Entomology, Hamburg, n. 123, p. 23-27, 1999.

KOCOUREK, F. et al. Effect of temperature on development rate and intrinsic rate of increase of Aphis gossypii reared on greenhouse cucumbers. Entomologia Experimentalis et Applicata, Dordrecht, v. 71, p. 59-64, 1994.

MATTHEWS, G. A.; TUNSTALL, J. P. Insect pests of cotton. Cambridge: Cab International, 1994. 593 p.

NEVES, O. S. et al. Cultura e adubação do algodoeiro. São Paulo: Instituto Brasileiro de Potassa, 1965. 567 p.

PASSOS, S. M. G. Algodão. Campinas: Instituto Campineiro de Ensino Agrícola, 1977. 424 p.

SOARES, J. J. Perspectivas do uso de variedades resistentes no manejo integrado de pragas do algodoeiro. Jaboticabal: FUNEP, 1999. 20 p.

SOGLIA, M. C. M.; BUENO, V. H. P.; SAMPAIO, M. V. Desenvolvimento e sobrevivência de Aphis gossypii Glover, 1877 (Hemiptera: Aphididae) em diferentes temperaturas e cultivares comerciais de crisântemo. Neotropical Entomology, Londrina, v. 31, n. 2, p. 211216, 2002.

TREACY, M. F. et al. Funtional response of a predator (Neuroptera: Chrysopidae) to bollworm (Lepidoptera: Noctuidae) eggs on smoothleaf, hirsute, and pilose cottons. Journal of Economic Entomology, College Park, v. 80, p. 376-379, 1987.

VENDRAMIM, J. D.; NAKANO, O. Aspectos biológicos de Aphis gossypii Glover, 1877 (Homoptera: Aphididae) em algodoeiro. Anais da Sociedade Entomológica do Brasil, Jaboticabal, v. 10, n. 2, p. 163-173, 1981.

WEATHERSBEE III, A. A.; HARDEE, D. D. Abundance of cotton aphids (Homoptera: Aphididae) and associated biological control agents on six cotton cultivars. Journal of Economic Entomology, College Park, v. 87, n. 1, p. 258-265, 1994. 